\title{
Superbranes and Super Born-Infeld Theories from Nonlinear Realizations
}

\author{
S. Bellucci ${ }^{a}$, E. Ivanov ${ }^{b}$ and S. Krivonos ${ }^{b}$

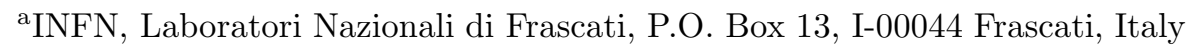 \\ bBogoliubov Laboratory of Theoretical Physics, JINR, 141980 Dubna, Moscow region, Russia
}

We describe, on a few instructive examples, a systematic way of deducing the superfield equations of motion of superbranes in the approach of partial breaking of global supersymmetry (PBGS) from the nonlinear-realizations formalism. For D-branes these equations simultaneously represent the appropriate supersymmetric Born-Infeld theories. We also discuss how to construct an off-shell superfield action for the $N=2, d=4$ Dirac-Born-Infeld theory corresponding to the partial supersymmetry breaking $N=4 \rightarrow N=2$ in $d=4$.

\section{INTRODUCTION}

There is a growing interest in applying the general method of nonlinear realizations [i] to systems with partial breaking of global supersymmetries (PBGS) [2], in particular, to the superbranes as a typical example of such systems (see, e.g., [3]13 and refs. therein). In this approach, the superbranes are described by the Goldstone superfields with the manifest linearly realized worldvolume supersymmetry and the nonlinearly realized rest of the original full target supersymmetry. The main difficulty one meets on this path is lacking of a systematic procedure for constructing the PBGS actions, as opposed, e.g., to the case of the total supersymmetry breaking [14, where the invariant actions can be constructed following the standard prescriptions of the nonlinear realizations method.

As a partial way out, it was proposed in 11 to use the nonlinear realizations approach to deduce the equations of motion for various types of superbranes in a manifestly covariant language of Cartan 1-forms. These equations are obtained as a direct covariantization of the free equations and irreducibility constraints for the Goldstone superfields. Miraculously, in many cases, while deriving such equations, there appears no need to incorporate the Goldstone superfields associated with the automorphism groups of the given supersymmetry, including the Lorentz group (though such superfields are in general required by the nonlinear realizations formalism). It proves enough to deal with the Goldstone superfields parametrizing spontaneously broken part of the translations and supertranslations. This circumstance greatly simplifies computations and allows one to get the superfield equations describing the superbranes wordvolume dynamics in a concise form.

One of the aims of the present talk is to exemplify this approach by the $D=4$ supermembrane and related to it via $T$-duality "space-filling" D2brane [7], as well as "space-filling" D3-brane (in $D=4)$ [5.6] and D3-branes in $D=6$ and $D=10$ [12]. These systems correspond, respectively, to the PBGS patterns $N=2 \rightarrow N=1$ in $d=3$ and $N=2 \rightarrow N=1, N=4 \rightarrow N=2$ and $N=8 \rightarrow N=4$ in $d=4$. In the case of D-branes the relevant Goldstone superfields encompass the abelian vector multiplets of unbroken (worldvolume) supersymmetry, with the Born-Infeld (BI) dynamics for the gauge field. Therefore in these cases the obtained superfield equations can be equivalently treated as the equations of $N=1$, $N=2$ and $N=4$ supersymmetric BI theories with hidden second supersymmetries.

As another topic, we discuss how to construct the full off-shell action for the $N=2$ BI theory with hidden $N=4$ supersymmetry in $d=4$, corresponding to the PBGS option $N=4 \rightarrow N=2$. While for the $N=2 \rightarrow N=1$ BI theory such 
an action was known 15,5], only partial results existed concerning an analogous $N=2 \mathrm{BI}$ action [16]17. We present, following the recent preprint [13], a systematic procedure for constructing $N=2$ BI action. It is based on embedding of $N=2$ vector multiplet into an infinitedimensional multiplet of the central-charge extended $N=4, d=4$ supersymmetry.

\section{2. $\mathrm{N}=1, \mathrm{D}=4$ SUPERMEMBRANE AND SPACE-FILLING D2-BRANE}

Let us start from the well known systems with partially broken global supersymmetries [4:7]. Our goal is to get the corresponding superfield equations of motion in terms of the worldvolume superfields starting from the nonlinear realization of the global supersymmetry group.

The supermembrane in $D=4$ spontaneously breaks half of the $N=1, D=4$ supersymmetry and one translation. Let us split the set of generators of $N=1 D=4$ Poincaré superalgebra (in the $d=3$ notation) into the unbroken $\left\{Q_{a}, P_{a b}\right\}$ and broken $\left\{S_{a}, Z\right\}$ ones $(a, b=1,2)$. The $d=3$ translation generator $P_{a b}=P_{b a}$ together with the generator $Z$ form the $D=4$ translation generator. The basic anticommutation relations read 1

$$
\left\{Q_{a}, Q_{b}\right\}=\left\{S_{a}, S_{b}\right\}=P_{a b},\left\{Q_{a}, S_{b}\right\}=\epsilon_{a b} Z \cdot(1)
$$

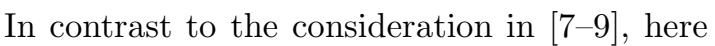
we prefer to deal with the nonlinear realization of the superalgebra (11) itself, ignoring all generators of the automorphisms of (11) (the spontaneously broken as well as unbroken ones), including those of $D=4$ Lorentz group $S O(1,3)$. Thus, we put all generators into the coset and associate the $N=1, d=3$ superspace coordinates $\left\{\theta^{a}, x^{a b}\right\}$ with $Q_{a}, P_{a b}$. The remaining coset parameters are Goldstone superfields, $\psi^{a} \equiv \psi^{a}(x, \theta), q \equiv q(x, \theta)$. A coset element $g$ is defined by

$g=e^{x^{a b} P_{a b}} e^{\theta^{a} Q_{a}} e^{q Z} e^{\psi^{a} S_{a}}$.

\footnotetext{
${ }^{1}$ Hereafter, we consider the spontaneously broken supersymmetry algebras modulo possible extra central-charge type terms which should be present in the full algebra of the corresponding Noether currents to evade the no-go theorem of ref. 18 along the lines of ref. [3].
}

As the next step, one constructs the Cartan 1forms

$$
\begin{aligned}
& g^{-1} d g=\omega_{Q}^{a} Q_{a}+\omega_{P}^{a b} P_{a b}+\omega_{Z} Z+\omega_{S}^{a} S_{a}, \\
& \omega_{P}^{a b}=d x^{a b}+\frac{1}{4} \theta^{(a} d \theta^{b)}+\frac{1}{4} \psi^{(a} d \psi^{b)}, \\
& \omega_{Z}=d q+\psi_{a} d \theta^{a}, \omega_{Q}^{a}=d \theta^{a}, \omega_{S}^{a}=d \psi^{a} .
\end{aligned}
$$

and define the covariant derivatives

$\mathcal{D}_{a b}=\left(E^{-1}\right)_{a b}^{c d} \partial_{c d}, \mathcal{D}_{a}=D_{a}+\frac{1}{2} \psi^{b} D_{a} \psi^{c} \mathcal{D}_{b c}$,

where

$$
\begin{aligned}
& D_{a}=\frac{\partial}{\partial \theta^{a}}+\frac{1}{2} \theta^{b} \partial_{a b},\left\{D_{a}, D_{b}\right\}=\partial_{a b} \\
& E_{a b}^{c d}=\frac{1}{2}\left(\delta_{a}^{c} \delta_{b}^{d}+\delta_{a}^{d} \delta_{b}^{c}\right)+\frac{1}{4}\left(\psi^{c} \partial_{a b} \psi^{d}+\psi^{d} \partial_{a b} \psi^{c}\right) .
\end{aligned}
$$

They obey the following algebra

$$
\begin{aligned}
& {\left[\mathcal{D}_{a b}, \mathcal{D}_{c d}\right]=-\mathcal{D}_{a b} \psi^{f} \mathcal{D}_{c d} \psi^{g} \mathcal{D}_{f g}} \\
& {\left[\mathcal{D}_{a b}, \mathcal{D}_{c}\right]=\mathcal{D}_{a b} \psi^{f} \mathcal{D}_{c} \psi^{g} \mathcal{D}_{f g}} \\
& \left\{\mathcal{D}_{a}, \mathcal{D}_{b}\right\}=\mathcal{D}_{a b}+\mathcal{D}_{a} \psi^{f} \mathcal{D}_{b} \psi^{g} \mathcal{D}_{f g}
\end{aligned}
$$

Not all of the above Goldstone superfields $\left\{q(x, \theta), \psi^{a}(x, \theta)\right\}$ must be treated as independent. Indeed, $\psi_{a}$ appears inside the form $\omega_{Z}$ linearly and so can be covariantly eliminated by the manifestly covariant constraint (inverse Higgs effect 19])

$$
\left.\omega_{Z}\right|_{d \theta}=0 \Rightarrow \psi_{a}=\mathcal{D}_{a} q
$$

where $\left.\right|_{d \theta}$ means the ordinary $d \theta$-projection of the form. Thus the superfield $q(x, \theta)$ is the only essential Goldstone superfield needed to present the partial spontaneous breaking $N=1, D=4 \Rightarrow$ $N=1, d=3$ within the coset scheme.

In order to get dynamical equations, we put additional, manifestly covariant constraints on the superfield $q(x, \theta)$. The idea is to covariantize the "flat" equations of motion. Namely, we replace the flat covariant derivatives in the standard equation of motion for the bosonic scalar superfield in $d=3$

$D^{a} D_{a} q=0$

by the covariant ones (5)

$\mathcal{D}^{a} \mathcal{D}_{a} q=0$. 
The equation (10) coincides with the equation of motion of the supermembrane in $D=4$ as it was presented in [7]. Thus, we conclude that, at least in this specific case, additional superfieldsparameters of the extended coset with all the automorphism symmetry generators included are auxiliary and can be dropped out if we are interested in the equations of motion only. Actually, it can be shown that (10) possesses the hidden covariance under the full $D=4$ Lorentz group.

As a straightforward application of the idea that the automorphism symmetries are irrelevant when deducing the equations of motion, let us consider the case of the "space-filling" D2-brane (i.e. having $N=1, d=3$ vector multiplet as its worldvolume one).

The superalgebra we start with is the algebra (11) without the central charge, $Z=0$. The coset element $g$ contains only one Goldstone superfield $\psi^{a}$ which now must be treated as the essential one, and the covariant derivatives coincide with (5). Bearing in mind to end up with the irreducible field content of $N=1, d=3$ vector multiplet, we are led to treat $\psi^{a}$ as the corresponding superfield strength and to find the appropriate covariantization of the flat irreducibility constraint and the equation of motion. In the flat case the $d=3$ vector multiplet is represented by a $N=1$ spinor superfield strength $\mu_{a}$ subjected to the Bianchi identity [20]:

$D^{a} \mu_{a}=0 \Rightarrow\left\{\begin{array}{l}D^{2} \mu_{a}=-\partial_{a b} \mu^{b} \\ \partial_{a b} D^{a} \mu^{b}=0\end{array}\right.$

This leaves in $\mu_{a}$ the first fermionic (Goldstone) component, together with the divergenceless vector $\left.F_{a b} \equiv D_{a} \mu_{b}\right|_{\theta=0}$ (i.e., just the gauge field strength). The equation of motion reads

$$
D^{2} \mu_{a}=0 \text {. }
$$

In accordance with our approach, we propose the following equations which should describe the D2brane:
(a) $\mathcal{D}^{a} \psi_{a}=0$
(b) $\mathcal{D}^{2} \psi_{a}=0$.

In order to see which kind of dynamics is encoded in (13), we considered it in the bosonic limit. We found that it amounts to the following equations for the vector $\left.V_{a b} \equiv \mathcal{D}_{a} \psi_{b}\right|_{\theta=0}$ :

$$
\left(\partial_{a c}+V_{a}^{m} V_{c}^{n} \partial_{m n}\right) V_{b}^{c}=0 .
$$

One can wonder how these nonlinear but polynomial equations can be related to the nonpolynomial BI theory which is just the bosonic core of the superfield D2-brane theory as was explicitly demonstrated in [7]. The trick is to rewrite the antisymmetric and symmetric parts of the equation (14) as follows:

$$
\begin{aligned}
& \partial_{a b}\left(\frac{V^{a b}}{2-V^{2}}\right)=0, \\
& \partial_{a c}\left(\frac{V_{b}^{c}}{2+V^{2}}\right)+\partial_{b c}\left(\frac{V_{a}^{c}}{2+V^{2}}\right)=0,
\end{aligned}
$$

where $V^{2} \equiv V^{m n} V_{m n}$. After passing to the "genuine" field strength

$F^{a b}=\frac{2 V^{a b}}{2-V^{2}} \Rightarrow \partial_{a b} F^{a b}=0$,

the equation of motion (16) takes the familiar BI form

$\partial_{a c}\left(\frac{F_{b}^{c}}{\sqrt{1+2 F^{2}}}\right)+\partial_{b c}\left(\frac{F_{a}^{c}}{\sqrt{1+2 F^{2}}}\right)=0$.

Thus we have proved that the bosonic part of our system (13) indeed coincides with the BI equations. One may explicitly show that the full equations (13) are equivalent to the worldvolume superfield equation following from the off-shell D2brane action given in [7.

\section{SPACE-FILLING D3-BRANE}

As another example we consider the spacefilling D3-brane in $d=4$. This system amounts to the PBGS pattern $N=2 \rightarrow N=1$ in $d=4$, with a nonlinear generalization of $N=1, d=4$ vector multiplet as the Goldstone multiplet [5, 6]. The off-shell superfield action for this system and the related equations of motion are known [5], but the latter have never been derived directly from the coset approach.

Our starting point is the $N=2, d=4$ Poincaré superalgebra without central charges:

$\left\{Q_{\alpha}, \bar{Q}_{\dot{\alpha}}\right\}=2 P_{\alpha \dot{\alpha}},\left\{S_{\alpha}, \bar{S}_{\dot{\alpha}}\right\}=2 P_{\alpha \dot{\alpha}}$. 
Assuming the $S_{\alpha}, \bar{S}_{\dot{\alpha}}$ supersymmetries to be spontaneously broken, we introduce the Goldstone superfields $\psi^{\alpha}(x, \theta, \bar{\theta}), \bar{\psi}^{\dot{\alpha}}(x, \theta, \bar{\theta})$ as the corresponding parameters in the following coset (we use the same notation as in [5])

$g=e^{i x^{\alpha \dot{\alpha}} P_{\alpha \dot{\alpha}}} e^{i \theta^{\alpha} Q_{\alpha}+i \bar{\theta}_{\dot{\alpha}} \bar{Q}^{\dot{\alpha}}} e^{i \psi^{\alpha} S_{\alpha}+i \bar{\psi}_{\dot{\alpha}} \bar{S}^{\dot{\alpha}}}$.

With the help of the Cartan forms

$$
\begin{aligned}
& g^{-1} d g= i \omega^{\alpha \dot{\alpha}} P_{\alpha \dot{\alpha}}+i \omega_{Q}^{\alpha} Q_{\alpha}+i \bar{\omega}_{Q \dot{\alpha}} \bar{Q}^{\dot{\alpha}} \\
&+i \omega_{S}^{\alpha} S_{\alpha}+i \bar{\omega}_{S \dot{\alpha}} \bar{S}^{\dot{\alpha}}, \\
& \omega^{\alpha \dot{\alpha}}= d x^{\alpha \dot{\alpha}}-i\left(\theta^{\alpha} d \bar{\theta}^{\dot{\alpha}}\right. \\
&\left.+\bar{\theta}^{\dot{\alpha}} d \theta^{\alpha}+\psi^{\alpha} d \bar{\psi}^{\dot{\alpha}}+\bar{\psi}^{\dot{\alpha}} d \psi^{\alpha}\right), \\
& \omega_{Q}^{\alpha}=d \theta^{\alpha}, \bar{\omega}_{Q}^{\dot{\alpha}}=d \bar{\theta}^{\dot{\alpha}}, \omega_{S}^{\alpha}=d \psi^{\alpha}, \bar{\omega}_{S}^{\dot{\alpha}}=d \bar{\psi}^{\dot{\alpha}},
\end{aligned}
$$

one can define the covariant derivatives

$$
\begin{aligned}
& \mathcal{D}_{\alpha}=D_{\alpha}-i\left(\bar{\psi}^{\dot{\beta}} D_{\alpha} \psi^{\beta}+\psi^{\beta} D_{\alpha} \bar{\psi}^{\dot{\beta}}\right) \mathcal{D}_{\beta \dot{\beta}}, \\
& \mathcal{D}_{\alpha \dot{\alpha}}=\left(E^{-1}\right)_{\alpha \dot{\alpha}}^{\beta \dot{\beta}} \partial_{\beta \dot{\beta}},
\end{aligned}
$$

where

$E_{\alpha \dot{\alpha}}^{\beta \dot{\beta}}=\delta_{\alpha}^{\beta} \delta_{\dot{\alpha}}^{\dot{\beta}}-i \psi^{\beta} \partial_{\alpha \dot{\alpha}} \bar{\psi}^{\dot{\beta}}-i \bar{\psi}^{\dot{\beta}} \partial_{\alpha \dot{\alpha}} \psi^{\beta}$,

and the flat covariant derivatives are defined as follows

$D_{\alpha}=\frac{\partial}{\partial \theta^{\alpha}}-i \bar{\theta}^{\dot{\alpha}} \partial_{\alpha \dot{\alpha}}, \bar{D}_{\dot{\alpha}}=-\frac{\partial}{\partial \bar{\theta}^{\dot{\alpha}}}+i \theta^{\alpha} \partial_{\alpha \dot{\alpha}}$

Now we are ready to write the covariant version of the constraints on $\psi^{\alpha}, \bar{\psi}^{\dot{\alpha}}$ which define the superbrane generalization of $N=1, d=4$ vector multiplet, together with the covariant equations of motion for this system.

As is well-known [21], the $N=1, d=4$ vector multiplet is described by a chiral $N=1$ field strength $W_{\alpha}$,

$\bar{D}_{\dot{\alpha}} W_{\alpha}=0, \quad D_{\alpha} \bar{W}_{\dot{\alpha}}=0$,

which satisfies the irreducibility constraint (Bianchi identity)

$D^{\alpha} W_{\alpha}+\bar{D}_{\dot{\alpha}} \bar{W}^{\dot{\alpha}}=0$.

The free equations of motion for the vector multiplet read

$D^{\alpha} W_{\alpha}-\bar{D}_{\dot{\alpha}} \bar{W}^{\dot{\alpha}}=0$.
It was shown in [5] that the chirality constraints (25) can be directly covariantized

$\overline{\mathcal{D}}_{\dot{\alpha}} \psi_{\alpha}=0, \quad \mathcal{D}_{\alpha} \bar{\psi}_{\dot{\alpha}}=0$.

These conditions are compatible with the algebra of the covariant derivatives (22). This algebra, with the constraints (28) taken into account, reads [5]

$$
\begin{aligned}
& \left\{\mathcal{D}_{\alpha}, \mathcal{D}_{\beta}\right\}=\left\{\overline{\mathcal{D}}_{\dot{\alpha}}, \overline{\mathcal{D}}_{\dot{\beta}}\right\}=0, \\
& \left\{\mathcal{D}_{\alpha}, \overline{\mathcal{D}}_{\dot{\beta}}\right\}=2 i \mathcal{D}_{\alpha \dot{\beta}}-2 i\left(\mathcal{D}_{\alpha} \psi^{\gamma} \overline{\mathcal{D}}_{\dot{\beta}} \bar{\psi}^{\dot{\gamma}}\right) \mathcal{D}_{\gamma \dot{\gamma}}, \\
& \left\{\mathcal{D}_{\alpha}, \mathcal{D}_{\gamma \dot{\gamma}}\right\}=-2 i\left(\mathcal{D}_{\alpha} \psi^{\beta} \mathcal{D}_{\gamma \dot{\gamma}} \bar{\psi}^{\dot{\beta}}\right) \mathcal{D}_{\beta \dot{\beta}} .
\end{aligned}
$$

The first two relations in (29) guarantee the consistency of the above nonlinear version of $N=$ $1, d=4$ chirality. They also imply, like in the flat case,

$(\mathcal{D})^{3}=(\overline{\mathcal{D}})^{3}=0$.

The second flat irreducibility constraint, eq.(26), is not so simple to covariantize. The straightforward generalization of (26),

$\mathcal{D}^{\alpha} \psi_{\alpha}+\overline{\mathcal{D}}_{\dot{\alpha}} \bar{\psi}^{\dot{\alpha}}=0$,

is contradictory. Let us apply the square $(\mathcal{D})^{2}$ to the left-hand side of (31). When hitting the first term in the sum, it yields zero in virtue of the property (30). However, it is not zero on the second term. To compensate for the resulting nonvanishing terms, and thus to achieve compatibility with the algebra (29) and its corollaries (30), one should modify (31) by some higher-order corrections [5].

Let us argue that the constraints (26) together with the equations of motion (27) can be straightforwardly covariantized as

$\mathcal{D}^{\alpha} \psi_{\alpha}=0, \quad \overline{\mathcal{D}}_{\dot{\alpha}} \bar{\psi}^{\dot{\alpha}}=0$.

Firstly, we note that no difficulties of the above kind related to the compatibility with the algebra (29) arise on the shell of eqs. (32). As a consequence of (32) and the first two relations in (29) we get

$\mathcal{D}^{2} \psi_{\alpha}=0, \quad \overline{\mathcal{D}}^{2} \bar{\psi}_{\dot{\alpha}}=0$.

This set is a nonlinear version of the well-known reality condition and the equation of motion for 
the auxiliary field of vector multiplet. Then, applying, e.g., $\mathcal{D}_{\alpha}$ to the second equation in (32) and making use of the chirality condition (28), we obtain the nonlinear version of the equation of motion for photino

$\mathcal{D}_{\alpha \dot{\alpha}} \bar{\psi}^{\dot{\alpha}}-\left(\mathcal{D}_{\alpha} \psi^{\gamma} \overline{\mathcal{D}}_{\dot{\alpha}} \bar{\psi}^{\dot{\gamma}}\right) \mathcal{D}_{\gamma \dot{\gamma}} \bar{\psi}^{\dot{\alpha}}=0$.

Acting on this equation by one more $\mathcal{D}_{\alpha}$ and taking advantage of the equations (33) we obtain the identity $0=0$, i.e. no new dynamical restrictions on $\psi^{\alpha}, \bar{\psi}^{\dot{\alpha}}$ arise. On the other hand, acting by $\overline{\mathcal{D}}_{\dot{\alpha}}$ produces a nonlinear generalization of the Maxwell equation. Further applying the covariant derivatives to this equation does not lead to new consequences. It can be also explicitly checked, in a few lowest orders in $\psi^{\alpha}, \bar{\psi}^{\dot{\alpha}}$, that the higher-order corrections to (31) found in [5] are vanishing on the shell of eqs. (32).

Thus the full set of equations describing the dynamics of the D3-brane supposedly consists of the generalized chirality constraint (28) and the equations (32). To prove its equivalence to the $N=1$ superfield description of D3-brane proposed in [5], recall that the latter is the $N=1$ supersymmetrization [15] of the $d=4 \mathrm{BI}$ action with one extra nonlinearly realized $N=1$ supersymmetry. So, let us consider the bosonic part of the proposed set of equations. Our superfields $\psi, \bar{\psi}$ contain the following bosonic components:

$\left.V^{\alpha \beta} \equiv \mathcal{D}^{\alpha} \psi^{\beta}\right|_{\theta=0}, \bar{V}^{\dot{\alpha} \dot{\beta}}=\left.\equiv \overline{\mathcal{D}}^{\dot{\alpha}} \bar{\psi}^{\dot{\beta}}\right|_{\theta=0}$,

which, owing to (32), obey the following simple equations

$$
\begin{aligned}
& \partial_{\alpha \dot{\alpha}} V^{\alpha \beta}-V_{\alpha}^{\gamma} \bar{V}_{\dot{\alpha}}^{\dot{\gamma}} \partial_{\gamma \dot{\gamma}} V^{\alpha \beta}=0, \\
& \partial_{\alpha \dot{\alpha}} \bar{V}^{\dot{\alpha} \dot{\beta}}-V_{\alpha}^{\gamma} \bar{V}_{\dot{\alpha}}^{\dot{\gamma}} \partial_{\gamma \dot{\gamma}} \bar{V}^{\dot{\alpha} \dot{\beta}}=0 .
\end{aligned}
$$

Like in the D2-brane case, in the equations (36) nothing reminds us of the BI equations. Nevertheless, it is possible to rewrite these equations in the standard BI form.

After some algebra, one can bring eqs.(36) into the following equivalent form

$$
\begin{aligned}
& \partial_{\beta \dot{\alpha}}\left(f V_{\alpha}^{\beta}\right)-\partial_{\alpha \dot{\beta}}\left(\bar{f} \bar{V}_{\dot{\alpha}}^{\dot{\beta}}\right)=0, \\
& \partial_{\beta \dot{\alpha}}\left(g V_{\alpha}^{\beta}\right)+\partial_{\alpha \dot{\beta}}\left(\bar{g} \bar{V}_{\dot{\alpha}}^{\dot{\beta}}\right)=0,
\end{aligned}
$$

where

$f=\frac{\bar{V}^{2}-2}{1-\frac{1}{4} V^{2} \bar{V}^{2}}, \quad g=\frac{\bar{V}^{2}+2}{1-\frac{1}{4} V^{2} \bar{V}^{2}}$.

After introducing the "genuine" field strengths

$F_{\alpha}^{\beta} \equiv \frac{1}{2 \sqrt{2}} f V_{\alpha}^{\beta}, \quad \bar{F}_{\dot{\alpha}}^{\dot{\beta}} \equiv \frac{1}{2 \sqrt{2}} \bar{f} \bar{V}_{\dot{\alpha}}^{\dot{\beta}}$,

first of eqs. (37) is recognized as the Bianchi identity

$\partial_{\beta \dot{\alpha}} F_{\alpha}^{\beta}-\partial_{\alpha \dot{\beta}} \bar{F}_{\dot{\alpha}}^{\dot{\beta}}=0$,

while the second one acquires the familiar form of the BI equation

$\partial_{\beta \dot{\alpha}}\left(\frac{1+A}{B} F_{\alpha}^{\beta}\right)+\partial_{\alpha \dot{\beta}}\left(\frac{1-A}{B} \bar{F}_{\dot{\alpha}}^{\dot{\beta}}\right)=0$,

where

$$
\begin{aligned}
& A=F^{2}-\bar{F}^{2}, \\
& B=\sqrt{\left(F^{2}-\bar{F}^{2}\right)^{2}-2\left(F^{2}+\bar{F}^{2}\right)+1} .
\end{aligned}
$$

Thus, in this new basis the action for our bosonic system is the BI action:

$S=\int d^{4} x \sqrt{\left(F^{2}-\bar{F}^{2}\right)^{2}-2\left(F^{2}+\bar{F}^{2}\right)+1}$.

Now the equivalence of the system (32) to the equations corresponding to the action of ref. [5] can be established like in the D2-brane case.

Note that at the full superfield level the redefinition (39) should correspond to passing from the Goldstone fermions $\psi_{\alpha}, \bar{\psi}_{\dot{\alpha}}$ which have the simple transformation properties in the nonlinear realization of $N=1, d=4$ supersymmetry but obey the nonlinear irreducibility constraints, to the ordinary Maxwell superfield strength $W_{\alpha}, \bar{W}_{\dot{\alpha}}$ defined by eqs. (25), (26). The nonlinear action in [5] was written just in terms of this latter object. The equivalent form (32) of the equations of motion and Bianchi identity is advantageous in that it is manifestly covariant under the second (hidden) supersymmetry, being constructed out of the covariant objects. 


\section{4. $\mathrm{N}=2$ BI THEORY WITH PARTIALLY BROKEN $\mathrm{N}=4$ SUPERSYMMETRY}

\subsection{Vector Goldstone multiplet for $N=4 \rightarrow N=2$}

Now we wish to derive the $N=2$ supersymmetric BI theory as a theory of the partial breaking of $N=4, d=4$ supersymmetry down to $N=2$ supersymmetry, with the vector $N=2$ multiplet as the Goldstone one. As follows from the field content of the latter, this kind of $N=2$ BI theory should amount to a static-gauge form of D3-brane in $D=6$.

To apply the nonlinear realizations techniques [11, we firstly need to specify $N=4, d=4$ supersymmetry to start with.

In the $N=2$ Maxwell theory, the basic object is a complex scalar $N=2$ off-shell superfield strength $\mathcal{W}$ which is chiral and satisfies one additional Bianchi identity:

(a) $\bar{D}_{\dot{\alpha} i} \mathcal{W}=0, D_{\alpha}^{i} \overline{\mathcal{W}}=0$,

(b) $D^{i k} \mathcal{W}=\bar{D}^{i k} \overline{\mathcal{W}}$.

Here,

$D_{\alpha}^{i}=\frac{\partial}{\partial \theta_{i}^{\alpha}}+i \bar{\theta}^{\dot{\alpha} i} \partial_{\alpha \dot{\alpha}}, \bar{D}_{\dot{\alpha} i}=-\frac{\partial}{\partial \bar{\theta}^{\dot{\alpha} i}}-i \theta_{i}^{\alpha} \partial_{\alpha \dot{\alpha}}$,

$D^{i j} \equiv D^{\alpha i} D_{\alpha}^{j}, \bar{D}^{i j} \equiv \bar{D}_{\dot{\alpha}}^{i} D^{\dot{\alpha} j},(i, j=1,2)$.

The superfield equation of motion for $\mathcal{W}$ reads

$D^{i k} \mathcal{W}+\bar{D}^{i k} \overline{\mathcal{W}}=0$

and, together with (44b), amounts to

$D^{i k} \mathcal{W}=\bar{D}^{i k} \overline{\mathcal{W}}=0$.

In order to incorporate an appropriate generalization of $\mathcal{W}$ into the nonlinear realization scheme as the Goldstone superfield, we need to have the proper bosonic generator in the algebra. The following central extension of $N=4, d=4$ Poincaré superalgebra suits this purpose

$$
\begin{aligned}
& \left\{Q_{\alpha}^{i}, \bar{Q}_{\dot{\alpha} j}\right\}=\left\{S_{\alpha}^{i}, \bar{S}_{\dot{\alpha} j}\right\}=2 \delta_{j}^{i} P_{\alpha \dot{\alpha}}, \\
& \left\{Q_{\alpha}^{i}, S_{\beta}^{j}\right\}=2 \varepsilon^{i j} \varepsilon_{\alpha \beta} Z, \\
& \left\{\bar{Q}_{\dot{\alpha} i}, \bar{Q}_{\dot{\beta} j}\right\}=-2 \varepsilon^{i j} \varepsilon_{\dot{\alpha} \dot{\beta}} \bar{Z},
\end{aligned}
$$

with all other (anti)commutators vanishing. Note an important feature that the complex central charge $Z$ appears in the crossing anticommutator, while the generators $(Q, \bar{Q})$ and $(S, \bar{S})$ on their own form two $N=2$ superalgebras without central charges. The full internal symmetry automorphism group of (49) (commuting with $P_{\alpha \dot{\alpha}}$ and $\left.Z\right)$ is $S O(5) \sim S p(2)$. Besides the manifest $R$-symmetry group $U(2)_{R}=S U(2)_{R} \times$ $U(1)_{R}$ acting as uniform rotations of the doublet indices of all spinor generators and the opposite phase transformations of the $S$ - and $Q$ generators, it also includes the 6-parameter quotient $S O(5) / U(2)_{R}$ transformations which properly rotate the generators $Q$ and $S$ through each other. The superalgebra (49) is a $d=4$ form of $N=(2,0)$ (or $N=(0,2))$ Poincaré superalgebra in $D=6$.

Let us now split the set of generators of the $N=4$ superalgebra (49) into the unbroken $\left\{Q_{\alpha}^{i}, \bar{Q}_{\dot{\alpha} j}, P_{\alpha \dot{\alpha}}\right\}$ and broken $\left\{S_{\alpha}^{i}, \bar{S}_{\dot{\alpha} j}, Z, \bar{Z}\right\}$ parts and define a coset element $g$ as:

$$
\begin{gathered}
g=\exp i\left(-x^{\alpha \dot{\alpha}} P_{\alpha \dot{\alpha}}+\theta_{i}^{\alpha} Q_{\alpha}^{i}+\bar{\theta}_{\dot{\alpha}}^{i} \bar{Q}_{i}^{\dot{\alpha}}\right) \\
\exp i\left(\psi_{i}^{\alpha} S_{\alpha}^{i}+\bar{\psi}_{\dot{\alpha}}^{i} \bar{S}_{i}^{\dot{\alpha}}\right) \exp i(W Z+\bar{W} \bar{Z}) .
\end{gathered}
$$

Acting on (50) from the left by various elements of the supergroup corresponding to (49), , one can find the transformation properties of the coset coordinates.

For the unbroken supersymmetry $\left(g_{0}=\exp i\left(-a^{\alpha \dot{\alpha}} P_{\alpha \dot{\alpha}}+\epsilon_{i}^{\alpha} Q_{\alpha}^{i}+\bar{\epsilon}_{\dot{\alpha}}^{i} \bar{Q}_{i}^{\dot{\alpha}}\right)\right)$ one has:

$$
\begin{aligned}
& \delta x^{\alpha \dot{\alpha}}=a^{\alpha \dot{\alpha}}-i\left(\epsilon_{i}^{\alpha} \bar{\theta}^{\dot{\alpha} i}+\bar{\epsilon}^{\dot{\alpha} i} \theta_{i}^{\alpha}\right), \\
& \delta \theta_{i}^{\alpha}=\epsilon_{i}^{\alpha}, \quad \delta \bar{\theta}_{\dot{\alpha}}^{i}=\bar{\epsilon}_{\dot{\alpha}}^{i} .
\end{aligned}
$$

Broken supersymmetry transformations $\left(g_{0}=\exp i\left(\eta_{i}^{\alpha} S_{\alpha}^{i}+\bar{\eta}_{\dot{\alpha}}^{i} \bar{S}_{i}^{\dot{\alpha}}\right)\right)$ are as follows:

$$
\begin{aligned}
& \delta x^{\alpha \dot{\alpha}}=-i\left(\eta_{i}^{\alpha} \bar{\psi}^{\dot{\alpha} i}+\bar{\eta}^{\dot{\alpha} i} \psi_{i}^{\alpha}\right), \\
& \delta \psi_{i}^{\alpha}=\eta_{i}^{\alpha}, \quad \delta \bar{\psi}_{\dot{\alpha}}^{i}=\bar{\eta}_{\dot{\alpha}}^{i}, \\
& \delta W=-2 i \eta_{i}^{\alpha} \theta_{\alpha}^{i}, \quad \delta \bar{W}=-2 i \bar{\eta}_{\dot{\alpha}}^{i} \bar{\theta}_{i}^{\dot{\alpha}} .
\end{aligned}
$$

Finally, the broken $Z, \bar{Z}$-translations $\left(g_{0}=\exp i(c Z+\bar{c} \bar{Z})\right) \mathrm{read}$

$\delta W=c, \quad \delta \bar{W}=\bar{c}$.

The next standard step is to define the leftinvariant Cartan 1-forms:

$\omega_{P}^{\alpha \dot{\alpha}}=d x^{\alpha \dot{\alpha}}-i\left(d \bar{\theta}^{\dot{\alpha} i} \theta_{i}^{\alpha}+d \theta_{i}^{\alpha} \bar{\theta}^{\dot{\alpha} i}\right)$ 


$$
\begin{aligned}
& -i\left(d \bar{\psi}^{\dot{\alpha} i} \psi_{i}^{\alpha}+d \psi_{i}^{\alpha} \bar{\psi}^{\dot{\alpha} i}\right) \\
\omega_{Q i}^{\alpha}= & d \theta_{i}^{\alpha}, \quad \omega_{S i}^{\alpha}=d \psi_{i}^{\alpha} \\
\omega_{Z}= & d W-2 i d \theta_{i}^{\alpha} \psi_{\alpha}^{i} .
\end{aligned}
$$

The covariant derivatives of some scalar $N=2$ superfield $\Phi$ are defined by expanding the differential $d \Phi$ over the covariant differentials of the $N=2$ superspace coordinates

$$
\begin{gathered}
d \Phi \equiv \omega_{P}^{\alpha \dot{\alpha}} \nabla_{\alpha \dot{\alpha}} \Phi+d \theta_{i}^{\alpha} \mathcal{D}_{\alpha}^{i} \Phi+d \bar{\theta}_{\dot{\alpha}}^{i} \overline{\mathcal{D}}_{i}^{\dot{\alpha}} \Phi \Rightarrow \\
\nabla_{\alpha \dot{\alpha}}=\left(E^{-1}\right)_{\alpha \dot{\alpha}}^{\beta \dot{\beta}} \partial_{\beta \dot{\beta}}, \\
E_{\alpha \dot{\alpha}}^{\beta \dot{\beta}} \equiv \delta_{\alpha}^{\beta} \delta_{\dot{\alpha}}^{\dot{\beta}}+i \psi_{i}^{\beta} \partial_{\alpha \dot{\alpha}} \bar{\psi}^{\dot{\beta} i}+i \bar{\psi}^{\dot{\beta} i} \partial_{\alpha \dot{\alpha}} \psi_{i}^{\beta}, \\
\mathcal{D}_{\alpha}^{i}=D_{\alpha}^{i}+i\left(\psi_{j}^{\beta} D_{\alpha}^{i} \bar{\psi}^{\dot{\beta} j}+\bar{\psi}^{\dot{\beta} j} D_{\alpha}^{i} \psi_{j}^{\beta}\right) \nabla_{\beta \dot{\beta}},
\end{gathered}
$$

where $D_{\alpha}^{i}, \bar{D}_{i \dot{\alpha}}$ are defined in (45).

As in previously studied examples, the Goldstone fermionic superfields $\psi_{\alpha}^{i}, \bar{\psi}_{\dot{\alpha} i}$ can be covariantly expressed in terms of the central-charge Goldstone superfields $\mathcal{W}, \overline{\mathcal{W}}$ by imposing the inverse Higgs constraints [19] on the central-charge Cartan 1-forms. In the present case these constraints are

$\left.\omega_{Z}\right|_{d \theta, d \bar{\theta}}=\left.\bar{\omega}_{Z}\right|_{d \theta, d \bar{\theta}}=0$,

where | means the covariant projections on the differentials of the spinor coordinates. These constraints amount to the sought expressions for the fermionic Goldstone superfields

$\psi_{\alpha}^{i}=-\frac{i}{2} \mathcal{D}_{\alpha}^{i} W, \quad \bar{\psi}_{\dot{\alpha} i}=-\frac{i}{2} \overline{\mathcal{D}}_{\dot{\alpha} i} \bar{W}$,

and, simultaneously, to the covariantization of the chirality conditions (44a)

$\overline{\mathcal{D}}_{\dot{\alpha} i} W=0, \quad \mathcal{D}_{\alpha}^{i} \bar{W}=0$.

Actually, eqs. (57) are highly nonlinear equations serving to express $\psi_{\alpha}^{i}, \bar{\psi}_{\dot{\alpha} i}$ in terms of $W, \bar{W}$ with making use of the definitions (55).

It is also straightforward to write the covariant generalization of the dynamical equation of the $N=2$ abelian vector multiplet (44), (48)

$\mathcal{D}^{\alpha(i} \mathcal{D}_{\alpha}^{j)} W=0, \quad \overline{\mathcal{D}}_{\dot{\alpha}}^{(i} \overline{\mathcal{D}}^{\dot{\alpha} j)} \bar{W}=0$.

The equations (58), (59) with the superfield Goldstone fermions eliminated by (57) constitute a manifestly covariant form of the superfield equations of motion of $N=2$ Dirac-BI theory with the second hidden nonlinearly realized $N=2$ supersymmetry. It closes, together with the manifest $N=2$ supersymmetry, on the $N=4$ supersymmetry $(49)$.

As a first step in proving this statement, let us show that the above system of equations reduces the component content of $W$ just to that of the on-shell $N=2$ vector multiplet. It is convenient to count the number of independent covariant superfield projections of $W, \bar{W}$.

At the dimensions $(-1)$ and $(-1 / 2)$ we find $W, \bar{W}$ and $\psi_{i \alpha}=-\frac{i}{2} \mathcal{D}_{i \alpha} W, \quad \bar{\psi}_{\dot{\alpha}}^{i}=\overline{\left(\psi_{i \alpha}\right)}=$ $-\frac{i}{2} \overline{\mathcal{D}}_{\dot{\alpha}}^{i} \bar{W}$, with a complex bosonic field and a doublet of gaugini as the lowest components.

At the dimension (0) we have, before employing (58), (59),

$$
\begin{aligned}
& \mathcal{D}_{\alpha}^{i} \psi_{\beta}^{j}=\varepsilon^{i j} f_{\alpha \beta}+i \varepsilon_{\alpha \beta} F^{(i j)}+F_{(\alpha \beta)}^{(i j)}, \\
& \overline{\mathcal{D}}_{\dot{\alpha} i} \psi_{j \alpha}=\varepsilon_{i j} X_{\alpha \dot{\alpha}}+X_{(i j) \alpha \dot{\alpha}}, \\
& f_{\alpha \beta} \equiv \epsilon_{\alpha \beta} A+i F_{\alpha \beta}, \bar{f}_{\dot{\alpha} \dot{\beta}}=\epsilon_{\dot{\alpha} \dot{\beta}} \bar{A}-i \bar{F}_{\dot{\alpha} \dot{\beta}}, \\
& f_{\beta}^{\alpha} f_{\alpha \gamma}=\epsilon_{\beta \gamma}\left(A^{2}-\frac{1}{2} F^{2}\right) .
\end{aligned}
$$

The dynamical equations (59) imply

$F^{(i j)}=\bar{F}^{(i j)}=0$.

The lowest component of these superfields is a nonlinear analog of the auxiliary field of the $N=$ 2 Maxwell theory.

Next, substituting the expressions (57) for the spinor Goldstone fermions in the 1.h.s. of eqs. (60) and making use of both (58) and (59), we represent these l.h.s. as

$$
\begin{aligned}
& \mathcal{D}_{\alpha}^{i} \psi_{\beta}^{j}=-\frac{i}{4}\left\{\mathcal{D}_{\alpha}^{i}, \mathcal{D}_{\beta}^{j}\right\} W+\frac{i}{4} \varepsilon^{i j} \mathcal{D}_{(\alpha}^{k} \mathcal{D}_{\beta) k} W, \\
& \overline{\mathcal{D}}_{\dot{\alpha} i} \psi_{\alpha}^{j}=-\frac{i}{2}\left\{\overline{\mathcal{D}}_{\dot{\alpha} i}, \mathcal{D}_{\alpha}^{j}\right\} W .
\end{aligned}
$$

Comparing (63) with the definition (60) (taking into account (62)), it is straightforward to show that the objects $F_{(\alpha \beta)}^{(i j)}, \bar{F}_{(i j)(\dot{\alpha} \dot{\beta})}, X_{(i j) \alpha \dot{\alpha}}$ and $\bar{X}_{\dot{\alpha} \alpha}^{(i j)}$ satisfy a system of homogeneous equations, such that the matrix of the coefficients in them is nonsingular at the origin $W=\bar{W}=0$. 
Thus these objects vanish as a consequence of the basic equations:

$F_{(\alpha \beta)}^{(i j)}=\bar{F}_{(i j)(\dot{\alpha} \dot{\beta})}=X_{(i j) \alpha \dot{\alpha}}=\bar{X}_{\dot{\alpha} \alpha}^{(i j)}=0$.

As a result, on shell we are left with the following superfield content:

$$
\begin{aligned}
& \mathcal{D}_{\alpha}^{i} \psi_{\beta}^{j}=\varepsilon^{i j} f_{\alpha \beta}, \quad \overline{\mathcal{D}}_{\dot{\alpha} i} \psi_{j \alpha}=\varepsilon_{i j} X_{\alpha \dot{\alpha}}, \\
& \overline{\mathcal{D}}_{\dot{\alpha} i} \bar{\psi}_{\dot{\beta} j}=-\varepsilon_{i j} \bar{f}_{\dot{\alpha} \dot{\beta}}, \quad \mathcal{D}_{\alpha}^{i} \bar{\psi}_{\dot{\alpha}}^{j}=\varepsilon^{i j} \bar{X}_{\dot{\alpha} \alpha} .
\end{aligned}
$$

The only new independent superfield at the dimension (0) is the complex one $F_{(\alpha \beta)}, \bar{F}_{(\dot{\alpha} \dot{\beta})}$, while $A, \bar{A}$ and $X_{\alpha \dot{\beta}}, \bar{X}_{\dot{\alpha} \beta}$ are algebraically expressed through it and other independent superfields as will be shown below. In the next Section we shall show that this superfield is related, by an equivalence field redefinition, to the Maxwell field strength obeying the BI equation of motion.

Substituting the explicit expressions for the anticommutators of covariant derivatives into (63) and again using (65) in both sides of (63), we finally obtain:

$$
\begin{aligned}
& X_{\alpha \dot{\alpha}}=\nabla_{\alpha \dot{\alpha}} W+\left(X_{\gamma \dot{\alpha}} \bar{X}_{\dot{\gamma} \alpha}+\bar{f}_{\dot{\alpha} \dot{\gamma}} f_{\alpha \gamma}\right) \nabla^{\gamma \dot{\gamma}} W, \\
& A=-\frac{1}{2} \bar{X}_{\dot{\gamma}}{ }^{\beta} f_{\beta \gamma} \nabla^{\gamma \dot{\gamma}} W
\end{aligned}
$$

It is easy to see that these algebraic equations indeed allow one to express $A, \bar{A}$ and $X_{\alpha \dot{\beta}}, \bar{X}_{\alpha \dot{\beta}}$ in terms of $F_{(\alpha \beta)}, \bar{F}_{(\dot{\alpha} \dot{\beta})}$ and $\nabla_{\alpha \dot{\alpha}} W, \nabla_{\alpha \dot{\alpha}} \bar{W}$ :

$$
\begin{aligned}
& X_{\alpha \dot{\alpha}}=\nabla_{\alpha \dot{\alpha}} W+\frac{1}{2}(\nabla W \cdot \nabla W) \nabla_{\alpha \dot{\alpha}} \bar{W}+\ldots, \\
& A=\frac{i}{2} F_{\gamma)}^{(\beta)} \nabla_{\beta \dot{\gamma}} \bar{W} \nabla^{\gamma \dot{\gamma}} W+\ldots
\end{aligned}
$$

Returning to the issue of extracting an irreducible set of covariant superfield projections of $W, \bar{W}$, it is easy to show that the further successive action by covariant spinor derivatives on (65) produces no new independent superfields. One obtains either the equations of motion (and Bianchi identities) for the independent basic superfields $W, \bar{W}, \psi_{i \alpha}, \bar{\psi}_{\dot{\alpha}}^{i}$ and $F_{(\alpha \beta)}, \bar{F}_{(\dot{\alpha} \dot{\beta})}$, or some composite superfields which are expressed through $x$-derivatives of the basic ones (or as some appropriate nonlinear functions of the basic superfields). The useful relations which essentially simplify the analysis are the following ones:

$$
\left\{\mathcal{D}_{\alpha}^{(i}, \overline{\mathcal{D}}_{\dot{\alpha}}^{j)}\right\}=\left\{\mathcal{D}_{\alpha}^{(i}, \mathcal{D}_{\beta}^{j)}\right\}=\left\{\overline{\mathcal{D}}_{\dot{\alpha}}^{(i}, \overline{\mathcal{D}}_{\dot{\alpha}}^{j)}\right\}=0
$$

These relations are the covariant version of the integrability conditions for the Grassmann harmonic $N=2$ analyticity [22]. Thus the nonlinear $W, \bar{W}$ background specified by the equations (57)-(59) respects the Grassmann harmonic analyticity which plays a fundamental role in $N=2, d=4$ theories.

Before going further, let us make a few comments.

First, the nonlinear realization setting we used, in order to deduce our equations (57)(59), drastically differs from the standard superspace differential-geometry setup of supersymmetric gauge theories (see, e.g., 21]). The starting point of the standard approach is the covariantization of the flat derivatives (spinor and vector) by the gauge-algebra valued connections with appropriate constraints on the relevant covariant superfield strengths. In our case (quite analogously to the $N=1, d=4$ and $N=1, d=3$ cases considered in Sect. 2 and 3) the covariant derivatives include no connection-type terms. Instead, they contain, in a highly non-linear manner, the Goldstone bosonic $N=2$ superfields $W, \bar{W}$. These quantities, after submitting them to the covariant constraints (57)-(59), turn out to be the nonlinear-realization counterparts of the $N=2$ Maxwell superfield strength. As we shall see, the Bianchi identities needed to pass to the gauge potentials are encoded in the set (57)-(59).

In our nonlinear system we cannot separate in a simple way the kinematical off-shell constraints from the dynamical on-shell ones. We could try to relax our system by lifting the basic dynamical equations (59) and retaining only the chirality condition (58) together with (57) and an appropriate covariantization of the constraint (44b). But in this case we immediately face the same difficulty as in the $N=2 \rightarrow N=1$ case: a naive covariantization of (44b) by replacing the flat spinor derivatives by the covariant ones proves to be not self-consistent. For selfconsistency, it should be properly modified order by order, without any clear guiding principle. No such a problem arises when the dynamical equations (59) are enforced. The terms modifying the naive covariantization of $(44 b)$ can be shown to vanish, as in the $N=2 \rightarrow N=1$ case [1]. 
Nevertheless, there exists a highly nonlinear field redefinition which relates the nonlinear superfield Goldstone strength $W, \bar{W}$ to its flat counterpart $\mathcal{W}, \overline{\mathcal{W}}$ satisfying the off-shell irreducibility conditions (44). In this frame it becomes possible to divide the kinematical and dynamical aspects of our system and to write the appropriate off-shell action giving rise to the dynamical equations, in a deep analogy with the $N=2 \rightarrow N=1$ case [5.6].

As the last comment, we note that all the fields of the multiplet comprised by $W, \bar{W}$, except for $F_{(\alpha \beta)}, \bar{F}_{(\dot{\alpha} \dot{\beta})}$, can be given a clear interpretation as Goldstone fields: $W|, \bar{W}|$ for the spontaneously broken central-charge shifts, $\psi_{\alpha}^{i}\left|, \bar{\psi}_{i \dot{\alpha}}\right|$ for the spontaneously broken $S$-supersymmetry transformations and $F^{(i j)}\left|, \bar{F}^{(i j)}\right|$ for the spontaneously broken $S O(5) / U(2)_{R}$ transformations.

\subsection{Bosonic equations of motion}

As the next important step in examining the superfield system (57)-(59), we inspect its bosonic sector. The set of bosonic equations can be obtained by acting on both sides of (65) by two covariantized spinor derivatives, using the relations (66) and omitting the fermions in the final expressions (which should contain only independent superfield projections and their $x$-derivatives). Instead of analyzing the bosonic sector in full generality, we specialize here to its two suggestive limits.

1. Vector fields limit. This limit amounts to

$\left.W\right|_{\theta=\bar{\theta}=0}=\left.\bar{W}\right|_{\theta=\bar{\theta}=0}=0$.

From eqs. (66) with all fermions omitted, one can see that (69) imply

$A=\bar{A}=X_{\alpha \dot{\alpha}}=\bar{X}_{\dot{\alpha} \alpha}=0$.

Thus, in this limit our superfields $W, \bar{W}$ contain only $F_{\alpha \beta}, \bar{F}_{\dot{\alpha} \dot{\beta}}$ as the bosonic components, which, owing to (59), obey the following simple equations

$\partial_{\alpha \dot{\alpha}} F^{\alpha \beta}-F_{\alpha}^{\gamma} \bar{F}_{\dot{\alpha}}^{\dot{\gamma}} \partial_{\gamma \dot{\gamma}} F^{\alpha \beta}=0$ and c.c. .

These equations coincide with eqs. (36) of the $N=2 \rightarrow N=1$ case. So they can be handled in the same way, i.e. split into the "true" Bianchi identities and "true" equations of motion which are the $d=4 \mathrm{BI}$ equations (41).
Thus the superfield system (57)-(59) encodes the BI equation, in accord with the statement that this system provides a supersymmetric extension of the latter.

2. Scalar fields limit. This limit corresponds to the reduction

$\left.\mathcal{D}_{(\alpha}^{i} \mathcal{D}_{\beta) i} W\right|_{\theta=\bar{\theta}=0}=\left.\overline{\mathcal{D}}_{(\dot{\alpha}}^{i} \overline{\mathcal{D}}_{\dot{\beta}) i} \bar{W}\right|_{\theta=\bar{\theta}=0}=0$.

From eqs. (66) one finds that the reduction conditions (72) imply

$A=0, X_{\alpha \dot{\alpha}}=\partial_{\alpha \dot{\alpha}} W+X_{\gamma \dot{\alpha}} \bar{X}_{\dot{\gamma} \alpha} \partial^{\gamma \dot{\gamma}} W$

(and c.c.), while the equations of motion following from (59) read

$\partial_{\dot{\alpha}}^{\alpha} X_{\alpha \dot{\beta}}+\bar{X}^{\dot{\gamma} \alpha} X_{\dot{\alpha}}^{\gamma} \partial_{\gamma \dot{\gamma}} X_{\alpha \dot{\beta}}=0$ and c.c. .

The system (73) can be easily solved

$X_{\alpha \dot{\alpha}}=\partial_{\alpha \dot{\alpha}} W+\frac{(\partial W)^{2}}{h} \partial_{\alpha \dot{\alpha}} \bar{W}$ and c.c.,

where

$$
\begin{aligned}
& h=1-B+\sqrt{(1-B)^{2}-C}, \\
& B=(\partial W \cdot \partial \bar{W}), C=(\partial W)^{2}(\partial \bar{W})^{2} .
\end{aligned}
$$

One can check that the symmetric parts of eqs. (74) are identically satisfied with (75) and (76). The trace part of (74) can be cast into the form:

$\partial_{\alpha \dot{\alpha}}\left(\frac{X^{\alpha \dot{\alpha}}+\frac{1}{2} X^{2} \bar{X}^{\dot{\alpha} \alpha}}{1-\frac{1}{4} X^{2} \bar{X}^{2}}\right)=0$ and c.c. .

Now, substituting (75), (76) in (77), one finds that the resulting form of these equations can be reproduced from the action

$S=\int d^{4} x\left(\sqrt{(1-B)^{2}-C}-1\right)$.

This action is the static-gauge form of the DiracNambu-Goto action of a 3-brane in $\mathrm{D}=6$.

Thus, we have shown that the system of our superfield equations (57)-(59) is self-consistent and gives a $N=2$ superextension of both the equations of $D=4$ BI theory and those of the staticgauge 3-brane in $D=6$, with the nonlinearly realized second $N=2$ supersymmetry. This justifies our claim that (57)-(59) are indeed a manifestly worldvolume supersymmetric form of the 
equations of D3-brane in $D=6$ and, simultaneously, of $N=2$ Born-Infeld theory. Similarly to the previous examples, the nonlinear realization approach yields the BI equations in a disguised form, with the Bianchi identities and dynamical equations mixed in a tricky way. At the same time, for the scalars we get the familiar static-gauge Nambu-Goto-type equations. This is in agreement with the fact that $W, \bar{W}$ undergo pure shifts under the action of the central charge generators $Z, \bar{Z}$, suggesting the interpretation of these superfields as the transverse brane coordinates conjugated to $Z, \bar{Z}$. These generators, in turn, can be interpreted as two extra components of the 6-momentum.

\subsection{Towards a formulation in terms of $\mathcal{W}, \overline{\mathcal{W}}$}

As was already mentioned, we expect that, like in the $N=1$ case [5,66], there should exist an equivalence transformation to a formulation in terms of the conventional $N=2$ Maxwell superfield strength $\mathcal{W}, \mathcal{W}$ defined by the off-shell constraints (44).

A systematic, though as yet iterative procedure to find such a field redefinition starts by passing to the standard chirality conditions $(44 a)$ from the covariantly-chiral ones (58). After some algebra, (58) can be brought to the form

$\bar{D}_{\dot{\alpha} i} R=0, \quad D_{\alpha}^{i} \bar{R}=0$,

where

$$
\begin{aligned}
R= & W+\frac{1}{2} \bar{W}(\partial W \cdot \partial W) \\
& +\frac{i}{4} D_{j}^{\gamma} W \bar{D}^{\dot{\gamma} j} \bar{W} \partial_{\gamma \dot{\gamma}} W+O\left(W^{5}\right) .
\end{aligned}
$$

Now we pass to the new superfields $\mathcal{W}, \overline{\mathcal{W}}$ with preserving the flat chirality

$$
\mathcal{W} \equiv R\left(1-\frac{1}{2} \bar{D}^{4} \bar{R}^{2}\right) \quad \text { and c.c. },
$$

$\bar{D}_{\dot{\alpha} i} \mathcal{W}=D_{\alpha}^{i} \overline{\mathcal{W}}=0$

where

$D^{4} \equiv \frac{1}{48} D^{\alpha i} D_{\alpha}^{j} D_{i}^{\beta} D_{\beta j}, \quad \bar{D}^{4}=\overline{\left(D^{4}\right)}$.
Up to the considered third order, in terms of these superfields eqs. (59) can be rewritten as

$$
\begin{aligned}
& D^{i j} \mathcal{W}=\bar{D}^{i j} \overline{\mathcal{W}} \\
& D^{i j}\left(\mathcal{W}+\mathcal{W} \bar{D}^{4} \overline{\mathcal{W}}^{2}\right) \\
& +\bar{D}^{i j}\left(\overline{\mathcal{W}}+\overline{\mathcal{W}} D^{4} \mathcal{W}^{2}\right)=0
\end{aligned}
$$

Eq. (83) is recognized as the Bianchi identity $(44 b)$, so $\mathcal{W}, \overline{\mathcal{W}}$ can be identified with the conventional $N=2$ vector multiplet superfield strength. Eq. (84) is then a nonlinear generalization of the standard free $N=2$ vector multiplet equation of motion (47). The transformation properties of $\mathcal{W}, \overline{\mathcal{W}}$ can be easily restored from (51)-(53) and the definitions 80, (81).

The above procedure is an $N=2$ superfield analog of separating Bianchi identities and dynamical equations for $F_{(\alpha \beta)}, \bar{F}_{(\dot{\alpha} \dot{\beta})}$. Though in the bosonic case we managed to find the appropriate field redefinition in a closed form, we are not aware of it in the full superfield case. Nonetheless, we can move a step further and find the relation between $W, \bar{W}$ and $\mathcal{W}, \mathcal{W}$, as well as the nonlinear dynamical equations for the latter, up to the fifth order. Then, using the transformation laws (51)(53), we can restore the hidden $S$-supersymmetry and $Z, \bar{Z}$ transformations up to the fourth order. In this approximation, the transformation laws and equations of motion read

$$
\begin{aligned}
& \delta \mathcal{W}=f-\frac{1}{2} \bar{D}^{4}\left(f \overline{\mathcal{A}}_{0}\right)+\frac{1}{4} \square\left(\bar{f} \mathcal{A}_{0}\right) \\
& +\frac{1}{4 i} \bar{D}^{i \dot{\alpha}} \bar{f} D_{i}^{\alpha} \partial_{\alpha \dot{\alpha}} \mathcal{A}_{0}, \quad \delta \overline{\mathcal{W}}=(\delta \mathcal{W})^{*} \\
& \mathcal{A}_{0}=\mathcal{W}^{2}\left(1+\frac{1}{2} \bar{D}^{4} \overline{\mathcal{W}}^{2}\right) \\
& f=c+2 i \eta^{i \alpha} \theta_{i \alpha} \\
& D^{i j} B+\bar{D}^{i j} \bar{B}=0 \\
& B=\mathcal{W}+\mathcal{W} \bar{D}^{4}\left(\overline{\mathcal{W}}^{2}+\overline{\mathcal{W}}^{2} D^{4} \mathcal{W}^{2}\right. \\
& \left.+\frac{1}{2} \overline{\mathcal{W}}^{2} \bar{D}^{4} \overline{\mathcal{W}}^{2}-\frac{1}{6} \mathcal{W} \square \overline{\mathcal{W}}^{3}\right)
\end{aligned}
$$

The hidden supersymmetry transformations, up to the third order, close on the $c, \bar{c}$ ones in the $\eta, \epsilon$ and $\bar{\eta}, \bar{\epsilon}$ sectors, and on the standard $d=4$ translations in the $\eta, \bar{\eta}$ sector. In the sectors $\eta, \eta$ and $\bar{\eta}, \bar{\eta}$ the transformations commute, as it should be. Note that (85) is already of the most general 
form compatible with the chirality conditions and Bianchi identity (44). So this form will be retained to any order, only the functions $\mathcal{A}_{0}, \overline{\mathcal{A}}_{0}$ will get additional contributions.

\section{OFF-SHELL ACTION OF THE $\mathrm{N}=2$ BI THEORY}

\subsection{Embedding $N=2$ vector multiplet into a linear $N=4$ multiplet}

In the previous Section we found the most general hidden supersymmetry transformation law of $\mathcal{W}, \overline{\mathcal{W}}(85)$ which is compatible with the defining constraints provided that the $N=2$ superfield function $\mathcal{A}_{0}$ is chiral

$\bar{D}_{\dot{\alpha} i} \mathcal{A}_{0}=0$.

By analogy with the $N=1$ construction of [5], in order to promote (85) to a linear (though still inhomogeneous) realization of the considered $N=4$ supersymmetry, it is natural to treat $\mathcal{A}_{0}$ as a new independent $N=2$ superfield constrained only by the chirality condition (89) and to try to define the transformation law of $\mathcal{A}_{0}$ under the $\eta, \bar{\eta}, c, \bar{c}$-transformations in such a way that the $N=2$ superfields $\mathcal{A}_{0}, \mathcal{W}, \overline{\mathcal{W}}$ form a closed set. Then, imposing a proper covariant constraint on these superfields one could hope to recover the structure (86) as the first terms in the solution to this constraint. In view of the covariance of this hypothetical constraint, the correct transformation law for $\mathcal{A}_{0}$ to the appropriate order can be reproduced by varying (86) according to the transformation law (85). Since we know $\mathcal{A}_{0}$ up to the 4th order, we can uniquely restore its transformation law up to the $3 \mathrm{~d}$ order. We explicitly find

$\delta \mathcal{A}_{0}=2 f \mathcal{W}+\frac{1}{4} \bar{f} \square \mathcal{A}_{1}+\frac{1}{4 i} \bar{D}^{i \dot{\alpha}} \bar{f} D_{i}^{\alpha} \partial_{\alpha \dot{\alpha}} \mathcal{A}_{1}$,

where

$\mathcal{A}_{1}=\frac{2}{3} \mathcal{W}^{3}+O\left(\mathcal{W}^{5}\right), \quad \bar{D}_{\dot{\alpha} i} \mathcal{A}_{1}=0$.

We observe the appearance of a new composite chiral superfield $\mathcal{A}_{1}$, and there is no way to avoid it in the transformation law (90). This is the crucial difference from the $N=1$ case of ref. 5 .6 where a similar reasoning led to a closed supermultiplet with only one extra $N=1$ superfield besides the $N=1$ Goldstone-Maxwell one (the resulting linear multiplet of $N=2$ supersymmetry is a $N=1$ superfield form of the $N=2$ vector multiplet with a modified transformation law 2324).

Thus, we are forced to incorporate a chiral superfield $\mathcal{A}_{1}$ as a new independent $N=2$ superfield component of the linear $N=4$ supermultiplet we are seeking. Inspecting the brackets of all these transformations suggests that the only possibility to achieve their closure in accord with the superalgebra (49) is to introduce an infinite sequence of chiral $N=2$ superfields and their antichiral conjugates $\mathcal{A}_{n}, \overline{\mathcal{A}}_{n}, n=0,1, \ldots$,

$\bar{D}_{\dot{\alpha} i} \mathcal{A}_{n}=0, D_{\alpha}^{i} \overline{\mathcal{A}}_{n}=0$,

with the following transformation laws:

$$
\begin{aligned}
\delta \mathcal{A}_{0} & =2 f \mathcal{W}+\frac{1}{4} \bar{f} \square \mathcal{A}_{1}+\frac{1}{4 i} \bar{D}^{i \dot{\alpha}} \bar{f} D_{i}^{\alpha} \partial_{\alpha \dot{\alpha}} \mathcal{A}_{1},(93) \\
\delta \mathcal{A}_{1} & =2 f \mathcal{A}_{0}+\frac{1}{4} \bar{f} \square \mathcal{A}_{2}+\frac{1}{4 i} \bar{D}^{i \dot{\alpha}} \bar{f} D_{i}^{\alpha} \partial_{\alpha \dot{\alpha}} \mathcal{A}_{2} \\
& \ldots \ldots \ldots . . \\
& \delta \mathcal{A}_{n}=2 f \mathcal{A}_{n-1}+\frac{1}{4} \bar{f} \square \mathcal{A}_{n+1} \\
& +\frac{1}{4 i} \bar{D}^{i \dot{\alpha}} \bar{f} D_{i}^{\alpha} \partial_{\alpha \dot{\alpha}} \mathcal{A}_{n+1}, \quad(n \geq 1) \\
\delta \overline{\mathcal{A}_{n}} & =\left(\delta \mathcal{A}_{n}\right)^{*} .
\end{aligned}
$$

It is a simple exercise to check that these transformations close off shell both among themselves and with those of the manifest $N=2$ supersymmetry just according to the superalgebra (49).

Realizing (formally) the central charge generators as derivatives in some extra complex "central-charge coordinate" $z$

$Z=\frac{i}{2} \frac{\partial}{\partial z}, \quad \bar{Z}=\frac{i}{2} \frac{\partial}{\partial \bar{z}}$,

and assuming all the involved $N=2$ superfields to be defined on a $z, \bar{z}$ extension of the standard $N=2$ superspace, it is instructive to rewrite the transformation laws under the $c, \bar{c}$ transformations as follows:

$\frac{\partial \mathcal{W}}{\partial z}=\left(1-\frac{1}{2} \bar{D}^{4} \overline{\mathcal{A}}_{0}\right), \quad \frac{\partial \mathcal{W}}{\partial \bar{z}}=\frac{1}{4} \square \mathcal{A}_{0} ，$ 


$$
\begin{array}{r}
\frac{\partial \mathcal{A}_{0}}{\partial z}=2 \mathcal{W}, \quad \frac{\partial \mathcal{A}_{0}}{\partial \bar{z}}=\frac{1}{4} \square \mathcal{A}_{1}, \\
\frac{\partial \mathcal{A}_{n}}{\partial z}=2 \mathcal{A}_{n-1}, \quad \frac{\partial \mathcal{A}_{n}}{\partial \bar{z}}=\frac{1}{4} \square \mathcal{A}_{n+1} .
\end{array}
$$

These relations imply, in particular,

$$
\begin{aligned}
& \left(\frac{\partial^{2}}{\partial z \partial \bar{z}}-\frac{1}{2} \square\right) \mathcal{W}=0, \\
& \left(\frac{\partial^{2}}{\partial z \partial \bar{z}}-\frac{1}{2} \square\right) \mathcal{A}_{n}=0 .
\end{aligned}
$$

If we regard $z, \bar{z}$ as the actual coordinates, which extend the $d=4$ Minkowski space to the $D=6$ one, the relations (99) mean that the constructed linear supermultiplet is on shell from the $D=6$ perspective. On the other hand, from the $d=4$ point of view this multiplet is off-shell, and the relations (96) - (98), (99) simply give a specific realization of the central charge generators $Z, \bar{Z}$ on its $N=2$ superfield components. In this sense this multiplet is similar to the previously known special $N=2, d=4$ and $N=$ $4, d=4$ supermultiplets, which are obtained from the on-shell multiplets in higher dimensions via non-trivial dimension reductions and inherit the higher-dimensional translation generators as nontrivially realized central charges in $d=4$ [25,26]. Since the superalgebra (49) is just a $d=4$ form of the $N=(2,0)$ (or $N=(0,2)) D=6$ Poincaré superalgebra, it is natural to think that the above supermultiplet has a $D=6$ origin and to try to reveal it. For the time being we prefer to treat the above infinite-dimensional representation in the pure $d=4$ framework as a linear realization of the partial spontaneous breaking of the centralcharge extended $N=4, d=4$ supersymmetry (49) to the standard $N=2$ supersymmetry.

\subsection{Invariant action}

In the approach proceeding from a linear realization of PBGS, the Goldstone superfield Lagrange density is, as a rule, a component of the same linear supermultiplet to which the relevant Goldstone superfield belongs. This is also true for the case under consideration. A good candidate for the chiral $N=2$ Lagrangian density is the superfield $\mathcal{A}_{0}$. Indeed, the "action"

$S=\int d^{4} x d^{4} \theta \mathcal{A}_{0}+\int d^{4} x d^{4} \bar{\theta} \overline{\mathcal{A}}_{0}$ is invariant with respect to the transformation (93) up to surface terms. With the interpretation of the central charge transformations as shifts with respect to the coordinates $z, \bar{z}$, the action (100) does not depend on these coordinates in virtue of eqs. (97), though the Lagrangian density can bear such a dependence.

It remains to define covariant constraints which would express $\mathcal{A}_{0}, \overline{\mathcal{A}}_{0}$ in terms of $\mathcal{W}, \overline{\mathcal{W}}$, with preserving the linear representation structure (85), (93), (94). Because an infinite number of $N=2$ superfields $\mathcal{A}_{n}$ is present in our case, there should exist an infinite set of constraints trading all these superfields for the basic Goldstone ones $\mathcal{W}, \overline{\mathcal{W}}$.

As a first step in finding these constraints let us note that the following expression:

$$
\begin{aligned}
\phi_{0}= & \mathcal{A}_{0}\left(1-\frac{1}{2} \bar{D}^{4} \overline{\mathcal{A}}_{0}\right)-\mathcal{W}^{2} \\
& -\sum_{k=1} \frac{(-1)^{k}}{2 \cdot 8^{k}} \mathcal{A}_{k} \square^{k} \bar{D}^{4} \overline{\mathcal{A}}_{k}
\end{aligned}
$$

is invariant, with respect to the $f$ part of the transformations (85), (93), (94). This leads us to choose

$\phi_{0}=0$

as our first constraint. For consistency with $N=$ 4 supersymmetry, the constraint (102) should be invariant with respect to the full transformations (85), (93), (94), with the $\bar{f}$ part taken into account as well. We shall firstly specialize to the $\bar{c}$ part of the $\bar{f}$ transformations. The requirement of the $\bar{c}$ covariance produces the new constraint

$$
\begin{aligned}
& \phi_{1}=\square \mathcal{A}_{1}+2\left(\mathcal{A}_{0} \square \mathcal{W}-\mathcal{W} \square \mathcal{A}_{0}\right) \\
& -\sum_{k=0} \frac{(-1)^{k}}{2 \cdot 8^{k}}\left(\square \mathcal{A}_{k+1} \square^{k} \bar{D}^{4} \overline{\mathcal{A}}_{k}\right. \\
& \left.-\mathcal{A}_{k+1} \square^{k+1} \bar{D}^{4} \overline{\mathcal{A}}_{k}\right)=0 .
\end{aligned}
$$

It is invariant under the $f$ transformations, but requiring it to be invariant also under the $\bar{c}$ part gives rise to the new constraint

$$
\begin{array}{r}
\phi_{2}=\square^{2} \mathcal{A}_{2}+2\left(\mathcal{A}_{0} \square^{2} \mathcal{A}_{0}-\square \mathcal{A}_{0} \square \mathcal{A}_{0}\right. \\
\left.+2 \square \mathcal{A}_{1} \square \mathcal{W}-\mathcal{A}_{1} \square^{2} \mathcal{W}-\mathcal{W} \square^{2} \mathcal{A}_{1}\right) \\
-\sum_{k=0} \frac{(-1)^{k}}{2 \cdot 8^{k}}\left(\square^{2} \mathcal{A}_{k+2} \square^{k} \bar{D}^{4} \overline{\mathcal{A}}_{k}\right.
\end{array}
$$


$\left.-2 \square \mathcal{A}_{k+2} \square^{k+1} \bar{D}^{4} \overline{\mathcal{A}}_{k}+\mathcal{A}_{k+2} \square^{k+2} \bar{D}^{4} \overline{\mathcal{A}}_{k}\right)=0$

and so on. The full infinite set of constraints is by construction invariant under the $f$ and $\bar{c}$ transformations. Indeed, using the relations (96)-(98) one may explicitly check that

$\frac{\partial \phi_{n}}{\partial z}=0, \quad \frac{\partial \phi_{n}}{\partial \bar{z}}=\frac{1}{4} \phi_{n+1}$,

so the full set of constraints is indeed closed.

The variation of the basic constraints with respect to the $\bar{f}$ transformations has the following general form:

$\delta \phi_{n}=\bar{\eta}^{i \dot{\alpha}} \bar{\theta}_{i \dot{\alpha}} \mathcal{B}_{n}+\bar{\eta}^{i \dot{\alpha}}\left(\mathcal{F}_{n}\right)_{i \dot{\alpha}}$.

Demanding this variation to vanish gives rise to the two sets of constraints

(a) $\mathcal{B}_{n}=0, \quad$ (b) $\left(\mathcal{F}_{n}\right)_{i \dot{\alpha}}=0$.

The constraints (106a) are easily recognized as those obtained above from the $\bar{c}$ covariance reasoning. One can show by explicit calculations that

$\bar{D}^{i \dot{\alpha}}\left(\mathcal{F}_{n}\right)_{j \dot{\beta}} \sim \delta_{j}^{i} \delta_{\dot{\beta}}^{\dot{\alpha}} \mathcal{B}_{n}$.

Thus the fermionic constraints (106 b) seem to be more fundamental. In order to prove that the basic fermionic constraints (106b) are actually equivalent to the bosonic ones (106a), one has to know the general solution to all constraints. For the time being we have explicitly checked this important property only for the iteration solution given below. Taking for granted that this is true in general, we can limit our attention to the type (a) constraints only. The constraints (101), (103) are just of this type.

At present we have no idea, how to explicitly solve the above infinite set of constraints and find a closed expression for the Lagrangian densities $\mathcal{A}_{0}, \overline{\mathcal{A}}_{0}$ similar to the one known in the $N=$ $2 \rightarrow N=1$ case $[5]$. What we are actually able to do, so far, is to restore a general solution by iterations. E.g., in order to restore the action up to the 8th order, we have to know the following orders in $\mathcal{A}_{k}$ :

$$
\begin{array}{r}
\mathcal{A}_{0}=\mathcal{W}^{2}+\mathcal{A}_{0}^{(4)}+\mathcal{A}_{0}^{(6)}+\mathcal{A}_{0}^{(8)}+\ldots, \\
\mathcal{A}_{1}=\mathcal{A}_{1}^{(3)}+\mathcal{A}_{1}^{(5)}+\mathcal{A}_{1}^{(7)}+\ldots \\
\mathcal{A}_{2}=\mathcal{A}_{2}^{(4)}+\mathcal{A}_{2}^{(6)}+\ldots, \quad \mathcal{A}_{3}=\mathcal{A}_{3}^{(5)}+\ldots
\end{array}
$$

These terms were found to have the following explicit structure:

$$
\begin{aligned}
& \mathcal{A}_{0}^{(4)}=\frac{1}{2} \mathcal{W}^{2} \bar{D}^{4} \overline{\mathcal{W}}^{2}, \\
& \mathcal{A}_{0}^{(6)}=\frac{1}{4} \bar{D}^{4}\left[\mathcal{W}^{2} \overline{\mathcal{W}}^{2}\left(D^{4} \mathcal{W}^{2}+\bar{D}^{4} \overline{\mathcal{W}}^{2}\right)\right. \\
& \left.-\frac{1}{9} \mathcal{W}^{3} \square \overline{\mathcal{W}}^{3}\right], \\
& \mathcal{A}_{0}^{(8)}=\frac{1}{8} \bar{D}^{4}\left[4 \mathcal{W}^{2} \overline{\mathcal{A}}_{0}^{(6)}+4 \overline{\mathcal{W}}^{2} \mathcal{A}_{0}^{(6)}\right. \\
& +\mathcal{W}^{2} \overline{\mathcal{W}}^{2} D^{4} \mathcal{W}^{2} \bar{D}^{4} \overline{\mathcal{W}}^{2}-\frac{2}{9} \mathcal{W}^{3} \square\left(\overline{\mathcal{W}}^{3} D^{4} \mathcal{W}^{2}\right) \\
& \left.-\frac{2}{9} \mathcal{W}^{3} \bar{D}^{4} \overline{\mathcal{W}}^{2} \square \overline{\mathcal{W}}^{3}+\frac{1}{144} \mathcal{W}^{4} \square^{2} \overline{\mathcal{W}}^{4}\right], \\
& \mathcal{A}_{1}^{(3)}=\frac{2}{3} \mathcal{W}^{3}, \quad \mathcal{A}_{1}^{(5)}=\frac{2}{3} \mathcal{W}^{3} \bar{D}^{4} \overline{\mathcal{W}}^{2}, \\
& \mathcal{A}_{1}^{(7)}=\bar{D}^{4}\left[\frac{1}{2} \mathcal{W}^{3} \overline{\mathcal{W}}^{2} \bar{D}^{4} \overline{\mathcal{W}}^{2}+\frac{1}{3} \mathcal{W}^{3} \overline{\mathcal{W}}^{2} D^{4} \mathcal{W}^{2}\right. \\
& \left.-\frac{1}{24} \mathcal{W}^{4} \square \overline{\mathcal{W}}^{3}\right], \\
& \mathcal{A}_{2}^{(4)}=\frac{1}{3} \mathcal{W}^{4}, \quad \mathcal{A}_{2}^{(6)}=\frac{1}{2} \mathcal{W}^{4} \bar{D}^{4} \overline{\mathcal{W}}^{2}, \\
& \mathcal{A}_{3}^{(5)}=\frac{2}{15} \mathcal{W}^{5} .
\end{aligned}
$$

Note that, despite the presence of growing powers of the operator $\square$ in our constraints, in each case the maximal power of $\square$ can be finally taken off from all the terms in the given constraint, leaving us with this maximal power of $\square$ acting on an expression which starts from the appropriate $\mathcal{A}_{n}$. Equating these final expressions to zero allows us to algebraically express all $\mathcal{A}_{n}$ in terms of $\mathcal{W}, \overline{\mathcal{W}}$ and derivatives of the latter. For example, for $\mathcal{A}_{3}^{(5)}$ we finally get the following equation:

$\square^{3} \mathcal{A}_{3}^{(5)}=\frac{2}{15} \square^{3} \mathcal{W}^{5} \Rightarrow \mathcal{A}_{3}^{(5)}=\frac{2}{15} \mathcal{W}^{5}$.

This procedure of taking off the degrees of $\square$ with discarding possible "zero modes" can be justified as follows: we are interested in an off-shell solution that preserves the manifest standard $N=2$ supersymmetry including the Poincaré covariance. This rules out possible on-shell zero modes as well as the presence of explicit $\theta$ 's or $x$ 's in the expressions which remain after taking off the appropriate powers of $\square$. It can be 
checked to any desirable order that these "reduced" constraints yield correct local expressions for the composite superfields $\mathcal{A}_{n}$, which prove to transform just in accordance with the original transformation rules (85), (93), (94). We have explicitly verified this for our iteration solution (109).

The explicit expression for the action, up to the 8th order in $\mathcal{W}, \overline{\mathcal{W}}$, reads

$$
\begin{aligned}
& S^{(8)}=\left(\int d^{4} x d^{4} \theta \mathcal{W}^{2}+\text { c.c. }\right) \\
& +\int d Z\left\{\mathcal{W}^{2} \overline{\mathcal{W}}^{2}\left[1+\frac{1}{2}\left(D^{4} \mathcal{W}^{2}+\bar{D}^{4} \overline{\mathcal{W}}^{2}\right)\right]\right. \\
& -\frac{1}{18} \mathcal{W}^{3} \square \overline{\mathcal{W}}^{3}+\frac{1}{4} \mathcal{W}^{2} \overline{\mathcal{W}}^{2}\left[\left(D^{4} \mathcal{W}^{2}+\bar{D}^{4} \overline{\mathcal{W}}^{2}\right)^{2}\right. \\
& \left.+D^{4} \mathcal{W}^{2} \bar{D}^{4} \overline{\mathcal{W}}^{2}\right]-\frac{1}{12} D^{4} \mathcal{W}^{2} \overline{\mathcal{W}}^{3} \square \mathcal{W}^{3} \\
& \left.-\frac{1}{12} \bar{D}^{4} \overline{\mathcal{W}}^{2} \mathcal{W}^{3} \square \overline{\mathcal{W}}^{3}+\frac{1}{576} \mathcal{W}^{4} \square^{2} \overline{\mathcal{W}}^{4}\right\} \cdot(111)
\end{aligned}
$$

This action, up to a slight difference in the notation, coincides with the action found by Kuzenko and Theisen 17] from the requirements of selfduality and invariance under nonlinear shifts of $\mathcal{W}, \overline{\mathcal{W}}$ (the $c, \bar{c}$ transformations in our notation). Let us point out that the structure of nonlinearities in the $c, \bar{c}$ transformations of $\mathcal{W}, \overline{\mathcal{W}}$ in our approach is uniquely fixed by the original $N=4$ supersymmetry transformations and the constraints imposed. The next, 10th order part of the $N=4$ invariant $N=2 \mathrm{BI}$ action can be easily restored from eqs. (109). Its explicit form looks not too enlightening, so we do not present it here. Actually, the action can be restored in this way to any order. It is of interest to find it in a closed form (if existing).

Finally, let us point out that after doing the $\theta$ integral, the pure Maxwell field strength part of the bosonic sector of the above action (and of the hypothetical complete action) comes entirely from the expansion of the standard Born-Infeld bosonic action. Just in this sense the above action is a particular $N=2$ extension of the bosonic BI action. The difference from the action of ref. [16] is just in higher-derivative terms with the $\square$ operators. These correction terms are crucial for the invariance under the hidden $N=2$ super- symmetry, and they drastically change, as compared to ref. [16], the structure of the bosonic action, both in the pure scalar fields sector and the mixed sector involving couplings between the Maxwell field strength and the scalar fields. By a reasoning of [11], the additional terms are just those needed for the existence of an equivalence field redefinition bringing the scalar fields action into the standard static-gauge Nambu-Goto form.

\section{6. $\mathrm{N}=4$ BI THEORY}

Finally, we derive the superfield equations of $N=4$ BI theory.

The $N=4, D=4$ Maxwell theory [28] is described by the covariant strength superfield $\mathcal{W}_{i j}=-\mathcal{W}_{j i},(i, j=1, \ldots, 4)$, satisfying the following independent constraints 27

$\overline{\mathcal{W}}^{i j} \equiv\left(\mathcal{W}_{i j}\right)^{*}=\frac{1}{2} \varepsilon^{i j k l} \mathcal{W}_{k l}$,

$D_{\alpha}^{k} \mathcal{W}_{i j}-\frac{1}{3}\left(\delta_{i}^{k} D_{\alpha}^{m} \mathcal{W}_{m j}-\delta_{j}^{k} D_{\alpha}^{m} \mathcal{W}_{m i}\right)=0$.

In contrast to the $N=2$ gauge theory, no offshell superfield formulation exists in the $N=4$ case: the constraints (112), (113) put the theory on shell.

As in the $N=2$ case, in order to construct a nonlinear generalization of (112), (113) one should firstly define the appropriate algebraic framework. It is given by the following central charge-extended $N=8, D=4$ Poincaré superalgebra:

$$
\begin{aligned}
& \left\{Q_{\alpha}^{i}, \bar{Q}_{\dot{\alpha} j}\right\}=2 \delta_{j}^{i} P_{\alpha \dot{\alpha}},\left\{S_{\alpha}^{i}, \bar{S}_{\dot{\alpha} j}\right\}=2 \delta_{j}^{i} P_{\alpha \dot{\alpha}} \\
& \left\{Q_{\alpha}^{i}, S_{\beta}^{j}\right\}=\varepsilon_{\alpha \beta} Z^{i j},\left\{\bar{Q}_{\dot{\alpha} i}, \bar{S}_{\dot{\beta} j}\right\}=\varepsilon_{\dot{\alpha} \dot{\beta}} \bar{Z}_{i j}, \\
& \bar{Z}_{i j}=\left(Z^{i j}\right)^{*}=\frac{1}{2} \varepsilon_{i j k l} Z^{k l}
\end{aligned}
$$

This is a $D=4$ notation for the type IIB Poincaré superalgebra in $D=10$.

We wish the $N=4, D=4$ supersymmetry $\left\{P_{\alpha \dot{\alpha}}, Q_{\alpha}^{i}, \bar{Q}_{\dot{\alpha} j}\right\}$ to remain unbroken, so we are led to introduce the Goldstone superfields

$$
\begin{aligned}
Z^{i j} & \Rightarrow W_{i j}(x, \theta, \bar{\theta}), \quad S_{\alpha}^{i} \Rightarrow \psi_{i}^{\alpha}(x, \theta, \bar{\theta}), \\
\bar{S}_{\dot{\alpha} j} & \Rightarrow \bar{\psi}_{i}^{\dot{\alpha}}(x, \theta, \bar{\theta}) .
\end{aligned}
$$


The reality property 1115$)$ automatically implies the constraint (112) for $W_{i j}$ :

$\bar{W}^{i j}=\frac{1}{2} \varepsilon^{i j k l} W_{k l}$.

On the coset element $g$

$$
\begin{aligned}
g= & \exp i\left(-x^{\alpha \dot{\alpha}} P_{\alpha \dot{\alpha}}+\theta_{i}^{\alpha} Q_{\alpha}^{i}+\bar{\theta}_{\dot{\alpha}}^{i} \bar{Q}_{i}^{\dot{\alpha}}\right) \\
& \exp i\left(\psi_{i}^{\alpha} S_{\alpha}^{i}+\bar{\psi}_{\dot{\alpha}}^{i} \bar{S}_{i}^{\dot{\alpha}}+W_{i j} Z^{i j}\right)
\end{aligned}
$$

one can realize the entire $N=8, D=4$ supersymmetry (114) by left shifts. The Cartan forms (except for the central charge one) and covariant derivatives formally coincide with (54)-(55), the indices $\{i, j\}$ now ranging from 1 to 4 . The central charge Cartan form reads:

$$
\begin{aligned}
\omega_{i j}^{Z}= & d W_{i j}+\frac{1}{2}\left(d \theta_{i}^{\alpha} \psi_{\alpha j}-d \theta_{j}^{\alpha} \psi_{\alpha i}\right. \\
& \left.+\varepsilon_{i j k l} d \bar{\theta}_{\dot{\alpha}}^{k} \bar{\psi}^{\dot{\alpha} l}\right) .
\end{aligned}
$$

By construction, it is covariant under all transformations of the $N=8, D=4$ Poincaré supergroup. The Goldstone superfields $\psi_{\alpha i}$ and $\bar{\psi}_{\dot{\alpha}}^{k}$ can be covariantly eliminated by the inverse Higgs procedure, as in the previous case. The proper constraint reads as follows:

$\left.\omega_{i j}^{Z}\right|_{d \theta, d \bar{\theta}}=0$.

It amounts to the following set of equations:

$$
\begin{aligned}
& \text { (a) } \mathcal{D}_{\alpha}^{k} W_{i j}+\frac{i}{2}\left(\delta_{i}^{k} \psi_{\alpha j}-\delta_{j}^{k} \psi_{\alpha i}\right)=0, \\
& \text { (b) } \overline{\mathcal{D}}_{k}^{\dot{\alpha}} W_{i j}+\frac{i}{2} \varepsilon_{i j k l} \bar{\psi}^{\dot{\alpha} l}=0,
\end{aligned}
$$

which are actually conjugated to each other in virtue of (117). We observe that, besides expressing the fermionic Goldstone superfields through the basic bosonic one $W_{i j}$ :

$\psi_{\alpha i}=-\frac{2 i}{3} \mathcal{D}_{\alpha}^{j} W_{i j}, \quad \bar{\psi}^{\dot{\alpha} i}=-\frac{2 i}{3} \overline{\mathcal{D}}_{j}^{\dot{\alpha}} \bar{W}^{i j}$,

eqs. (121) impose the nonlinear constraint

$\mathcal{D}_{\alpha}^{k} W_{i j}-\frac{1}{3}\left(\delta_{i}^{k} \mathcal{D}_{\alpha}^{m} W_{m j}-\delta_{j}^{k} \mathcal{D}_{\alpha}^{m} W_{m i}\right)=0$

(and its conjugate). This is the sought nonlinear generalization of (113).

It is straightforward to show that eq. 123. implies the disguised form of the BI equation for the nonlinear analog of the abelian gauge field strength. For the six physical bosonic fields $W_{i j} \mid$ we expect the equations corresponding to the static-gauge of 3-brane in $D=10$ to hold. Thus eqs. (117), (123) plausibly give a manifestly worldvolume supersymmetric description of D3brane in a flat $D=10$ Minkowski background. No simple off-shell action can be constructed in this case, since such an action is unknown even for the free $N=4$ Maxwell theory. But even the construction of the physical fields component action for this $N=4$ BI theory is of considerable interest. We hope to study this system in more detail elsewhere.

\section{CONCLUSIONS}

We presented a systematic approach to deducing the dynamics of superbranes from nonlinear realizations of the appropriate PBGS patterns in a way manifestly covariant under the worldvolume supersymmetry. In the case of D-branes the corresponding equations simultaneously describe the appropriate superextensions of BI theory. We also proposed a procedure for constructing the off-shell superfield action of $N=2, d=4$ BI theory associated with the PBGS pattern $N=4 \rightarrow$ $N=2$ (D3-brane in $D=6$ ).

Among the problems for further study let us distinguish generalizing the above consideration to non-abelian super BI theories and incorporating non-trivial curved backgrounds into the PBGS framework. As a first important step in tackling the second task it would be tempting to find PBGS formulations of superbranes with the $A d S_{n} \times S^{m}$ bosonic parts. As a more technical problem, we finally mention finding out the precise correspondence of the PBGS examples considered here with the superembedding approach to superbranes 29] along the lines of refs. [30], [31], 32]. In 331, 32] such a correspondence has been established for the $N=1, D=4$ supermembrane and space-filling D3-brane.

\section{Acknowledgements}

We are grateful to Eric Bergshoeff, Sergio Ferrara, Sergei Ketov, Sergei Kuzenko, Dmitri 
Sorokin and Mario Tonin for useful discussions. This work was supported in part by the Fondo Affari Internazionali Convenzione Particellare INFN-JINR, grants RFBR-CNRS 98-0222034, RFBR-DFG-99-02-04022, RFBR 99-0218417 and NATO Grant PST.CLG 974874.

\section{REFERENCES}

1. S. Coleman, J. Wess, B. Zumino, Phys. Rev. 177 (1969) 2239; C. Callan, S. Coleman, J. Wess, B. Zumino, ibid. 177 (1969) 2247; D.V. Volkov, Sov. J. Part. Nucl. 4 (1973) 3; V.I. Ogievetsky, In: Proceedings of Xth Winter School of Theoretical Physics in Karpacz, Acta Universitatis Wratislaviensis, Vol 207, p. 227, Wroclaw, 1974.

2. J. Bagger, J. Wess, Phys. Lett. B 138 (1984) 105.

3. J. Hughes, J. Liu, J. Polchinski, Phys. Lett. B 180 (1986) 370; J. Hughes, J. Polchinski, Nucl. Phys. B 278 (1986) 147.

4. A.Achucarro, J. Gauntlett, K. Itoh, P.K. Townsend, Nucl. Phys. B 314 (1989) 129.

5. J. Bagger, A. Galperin, Phys. Rev. D 55 (1997) 1091.

6. M. Roček, A. Tseytlin, Phys. Rev. D 59 (1999) 106001.

7. E. Ivanov, S. Krivonos, Phys. Lett. B 453 (1999) 237.

8. S. Bellucci, E. Ivanov, S. Krivonos, Phys. Lett. B 460 (1999) 348.

9. S. Bellucci, E. Ivanov, S. Krivonos, In: Proceedings of 32nd International Symposium Ahrenshoop, Septemeber 1 - 5, 1998, Buckow, Germany, Fortsch. Phys. 48 (2000) 19.

10. P. West, "Automorphisms, Non-linear Realizations and Branes", hep-th/0001216.

11. S. Bellucci, E. Ivanov, S. Krivonos, Phys. Lett. B 482 (2000) 233.

12. S. Bellucci, E. Ivanov, S. Krivonos, Phys. Lett. B 502 (2001) 279.

13. S. Bellucci, E. Ivanov, S. Krivonos, "Towards the complete $N=2$ superfield Born-Infeld action with partially broken $N=4$ supersymmetry", hep-th/0101195.

14. D.V. Volkov, V.P. Akulov, Phys. Lett. B 46 (1973) 109.
15. S. Cecotti, S. Ferrara, Phys. Lett. B 187 (1987) 335.

16. S. Ketov, Mod. Phys. Lett. A 14 (1999) 501; Class. Quant. Grav. 17 (2000) L91.

17. S.M. Kuzenko, S. Theisen, "Nonlinear SelfDuality and Supersymmetry", LMU-TPW00-19, July 2000, hep-th/0007231; JHEP 0003 (2000) 034.

18. E. Witten, Nucl. Phys. B 188 (1981) 513.

19. E.A. Ivanov, V.I. Ogievetsky, Teor. Mat. Fiz. 25 (1975) 164.

20. S.J. Gates, M.T. Grisaru, M. Roček, W. Siegel, Superspace, Benjamin/ Cummings, Reading, Massachusetts, 1983.

21. J. Wess, J. Bagger, "Supersymmetry and Supergravity", Princeton University Press, Princeton, 1992.

22. A. Galperin, E. Ivanov, S. Kalitzin, V. Ogievetsky, E. Sokatchev, Class. Quant. Grav. 1 (1984) 469.

23. I. Antoniadis, H. Partouche, T.R. Taylor, Phys. Lett. B 372 (1996) 83.

24. E.A. Ivanov, B.M. Zupnik, Yadern. Fiz. 62 (1999) 1110 (hep-th/9710236).

25. M.F. Sohnius, Nucl. Phys. B 138 (1978) 109.

26. M.F. Sohnius, K.S. Stelle, P.C. West, Phys. Lett. 92 B (1980) 123; Nucl. Phys. B173 (1980) 127.

27. M.F. Sohnius, Nucl. Phys. B 136 (1978) 461.

28. F. Gliozzi, J. Scherk, D. Olive, Nucl. Phys. B 122 (1977) 253.

29. D. Sorokin, Phys. Reports 329 (2000) 1.

30. T. Adawi, M. Cederwall, U. Gran, M. Holm, B.E. Nilsson, Int. J. Mod. Phys. A 13 (1998) 4691.

31. P. Pasti, D. Sorokin, M. Tonin, Nucl. Phys. B 591 (2000) 109; "Geometrical aspects of superbrane dynamics", hep-th/0011020.

32. I. Bandos, P. Pasti, A. Pokotilov, D. Sorokin, M. Tonin, "Space Filling Dirichlet 3-Brane in $N=2, D=4$ Superspace", contribution to these Proceedings, hep-th/0103152. 